\title{
LA ECONOMÍA POLÍTICA DE LA COMUNICACIÓN Y EL PENSAMIENTO CRÍTICO LATINOAMERICANO ENTREVISTA A CÉSAR BOLAÑO'
}

\section{POLITICAL ECONOMY IN COMMUNICATION AND LATINAMERICAN CRITICAL THINKING AN INTERVIEW WITH CÉSAR BOLAÑO}

\section{A ECONOMIA POĹTIICA DA COMUNICAÇ̃̃o E O PENSAMENTO CRÍTICO LATINOAMERICANO ENTREVISTA A CÉSAR BOLAÑO}

\section{Páginas Diego García Ramírez}

122-126 garcia.ramirez.diego@gmail.com

Antropólogo de la Universidad de Antioquia. Magister en Comunicación de la Pontificia Universidad Javeriana. Doctorando en Comunicación y Cultura de la Universidad Federal de Rio de Janeiro. Docente de la Escuela de Ciencias de la Comunicación en la Universidad Sergio Arboleda, Bogotá Colombia.

Entrevista realizada el 5 de octubre de 2016 en el marco del XII Congreso de la Asociación Latinoamérica de Investigadores en Comunicación. Ciudad de México, México. 
En años recientes, la Economía Política de la Comunicación (EPC) ha ganado una visibilidad con la que no contaba hace unas décadas. Este resurgir nace por los aportes que ha brindado para analizar los cambios y transformaciones ocurridas en la comunicación y la cultura en la era digital, además, con el aumento de la participación de las industrias culturales en la economía. Sin embargo, en Latinoamérica aún son pocos los trabajos e investigaciones que se realizan desde los postulados de la EPC. Para conocer más sobre la Economía Política de la Comunicación conversamos con el brasilero César Bolaño, uno de los autores más destacados en este campo en nuestro contexto, quien habló acerca de la historia, los debates y aportes de la EPC al pensamiento comunicacional latinoamericano.

\section{¿Qué es la Economía Política de la Comunicación?}

Es difícil dar una definición sucinta. Pero recientemente la definí como aquel conocimiento crítico que se produce en relación a la comunicación-en la de la vieja crítica realizada por Marx-. Ahora, esa es una definición personal, porque si buscas otros autores tal vez encuentres definiciones un poco diferentes.

\section{¿La Economía Política de la Comunicación es poco conocida 0 poco trabajada en Latinoamérica?}

Hoy es mucho más conocida que en el pasado. Creo que había una escuela crítica importante en Latinoamérica, la de las teorías de la dependencia, por ejemplo. Las críticas a esa escuela acabaron por poner en relieve los Estudios Culturales, la economía política hace también la crítica a estos enfoques anteriores, pero en una perspectiva más interna. Sin embargo, durante mucho tiempo quedó olvidada y a partir de determinado momento comenzó a crecer, por eso hoy está mucho más en evidencia que hace 10 años.

\section{¿Cuáles son los puntos de convergencia y divergencia entre la economía política y los estudios culturales?}

Ese es un tema complejo, hay muchas críticas que se han hecho al respecto. Creo que los estudios culturales latinoamericanos han cumplido un papel importante en cuanto a la crítica a las perspectivas anteriores, básicamente por defender una perspectiva más relacionada con los públicos, la capacidad de acción de los públicos; pero con esto muchas veces dejó de lado la otra determinación: la economía política.

En la versión que yo defiendo se analiza esta contradicción, jamás dejo de pensar el tema en términos de contradicción y en una perspectiva marxista. Los estudios culturales tenían una perspectiva marxista, pero en los años 90 del siglo pasado adoptan referentes postmodernistas muy complejos. Por otro lado, el problema principal que tuvieron fue el énfasis en la mediación desde el punto de vista de la recepción. Mientras que yo, por ejemplo, defino la mediación en términos de contradicción; o sea, la mediación que hace la industria cultural, los grandes medios de comunicación a través del trabajo creativo. Esto sería encauzar otra vez el problema en el sentido de la lucha de clases, cosa que los estudios culturales dejaron de lado hace mucho tiempo.

\section{¿Se puede hablar de una perspectiva}

latinoamericana de la economía política?

No sé si se puede decir "la perspectiva latinoamericana”, pero la formación de los economistas políticos en América Latina es distinta a la de otros lugares; por ejemplo, yo parto en mi análisis directamente de la lectura de Marx, como también hacen los franceses y otros; pero el debate académico que yo he conformado, los problemas que me fueron puestos y que asumí como problemas teóricos, están dentro de un marco cultural propio. Hablo del caso brasilero, no sé si en Argentina o México sea distinto, pero por lo general hay una cultura académica latinoamericana que se distingue de las otras. Además, nosotros tenemos la obligación de mostrar eso, dejar esto claro, y creo que eso es un reto para mi generación, porque es muy preocupante que nuevas generaciones que hablan inglés, y se comunican en esa lengua, desconozcan el pensamiento crítico que se hizo en América Latina. Economía política latinoamericana no es una definición estática, sino que es un reto. 
Es decir, ¿la perspectiva latinoamericana debe entrar en la discusión global de la economía política?

En esa discusión global se trata de recuperar la contribución de Latinoamérica, porque si vas a Brasil, por ejemplo, vas a ver que los estudiantes que están en las escuelas de comunicación, que comienzan a estudiar economía política, conocen más el libro de Vicent Mosco que el mío. Es una distorsión porque Mosco es fantástico, muy interesante, pero no está empapado de la problemática, de la cultura y referencias bibliográficas que he tenido en la escuela de comunicación, de la escuela de economía y la sociología brasileña.

¿Qué papel han jugado ALAIC ${ }^{2}$ y la ULEPICC ${ }^{3}$ para congregar el pensamiento latinoamericano?

Ese fue nuestro reto desde el principio. Yo fui el fundador del primer grupo de economía política en América Latina, que fue el de INTERCOM ${ }^{4}$, poco tiempo después el de alaic, y en adelante hemos creado una serie de instituciones formales e informales. ulepicc fue un proyecto entre un grupo de personas que compartían esa perspectiva, la del pensamiento latinoamericano, la economía política crítica, el pensamiento marxista en el interior de la comunicación y a través de determinadas estrategias de acción en el campo. Creo que eso fue una conquista, pero las conquistas no fueron hechas para que uno se duerma. Estamos enfrentando ahora otros retos muy importantes y otros pensamientos porque la lucha epistemológica sigue.

Usted conoce bien el caso brasileño, pero ¿tiene idea si en Latinoamérica en los programas de pregrado y posgrado se familiariza a los estudiantes con la Economía política?

En Brasil muy poco, yo creo que en Latinoamérica también muy poco, pero más que en Brasil. Este

2 Asociación Latinoamericana de Investigadores en Comunicación.

3 Unión Latina de Economía Política de la Información, la Comunicación y la Cultura.

4 Sociedade Brasileira de Estudos Interdisciplinares da Comunicação. es un problema que nosotros estamos enfrentado, porque desde el momento que se formaron los grupos de Intercom, de alaic, la ulepicc, estuvimos actuando muy fuerte sobre las asociaciones, pero mientras esto no sea reconocido académicamente en las escuelas será un problema. En Brasil está ocurriendo en este momento, apenas está comenzando; dos, tres o cuatro universidades están enseñando la EPc en pregrado. Yo creo que en América Latina hay más, en Europa hay mucha más economía política, otras economías políticas, en Francia y en otros países. Esto apenas comienza en Brasil y en Latinoamérica, yo creo que este es el problema, porque comienza muchas veces por la importación del pensamiento europeo y norteamericano, y no estoy diciendo que sea malo, pero hay que recuperar nuestras fuentes.

El surgimiento de la EPC en Latinoamérica no es muy posterior a otros, adicionalmente, surge sin conocimiento de otros o con un conocimiento a veces muy parcial. En Brasil, México, Argentina, Chile, hay precursores de este pensamiento; gente que conoce el debate latinoamericano de las comunicaciones y que conoce a Marx y que quiere hacer esta relación, y muchos de estos autores no son conocidos por los propios economistas políticos latinoamericanos jóvenes.

Ese fue uno de los retos que nosotros asumimos como fundamentales: recuperar esa forma de pensamiento crítico.

Dejando atrás un poco la historia de la EPC en América Latina, ¿qué tiene para decir la EPC en la era digital? porque parece que con la digitalización y la convergencia tecnológica, la EPc toma importancia para pensar nuevamente la función económica de los medios de comunicación.

Sí, es interesante. Lo que pasa es que hubo dos momentos en ese proceso: un momento en que se decía "esto ya no tiene ningún sentido"; por ejemplo, Marx no explica ya nada porque se cayó el muro de Berlín, cosas que no tienen nada que ver. Otro momento de ataques muy fuerte al pensamiento crítico, 
sobre todo desde el pensamiento posmoderno y el neoliberalismo. Hasta que se hicieron evidentes las contradicciones del sistema, y no solamente las contradicciones económicas, sino también en materia de comunicación, la internet, por ejemplo. Nosotros peleamos por mucho tiempo con gente que creía que la internet nos iba a liberar, que era un instrumento de democratización, hasta que llegó Edward Snowden y ahí la gente se dio cuenta de lo que nosotros estábamos diciendo desde los años 90: "internet es un instrumento de control social"; entonces la misma crítica que nosotros hemos hecho a las industrias culturales hay que hacérsela a internet y es más difícil.

Ahora la gente mira la EPC de otra forma porque sabe lo coherente que hemos sido, en la época en que esto no estaba de moda y que era un problema, entonces ahora vienen a buscar explicaciones, porque la EPc tiene la capacidad al ser parte de un paradigma materialista histórico, tiene la capacidad de dar una versión muy completa y amplia del problema, para entender cómo el tema de la comunicación se inserta en los movimientos globales del capital.

\section{Usted que habla de contradicciones} ¿también hay contradicciones conceptuales? ahora se habla de economía e industrias creativas, industrias del entretenimiento...

Esos son términos en disputa, la idea de industria creativa es una idea que viene pareja al neoliberalismo. Son términos en disputa, a mí no me importa que se diga economía creativa o industrias creativas ¿por qué? porque hay una contradicción específica en esto, ya no se trata de cualquier trabajo sino de trabajo creativo ¿cómo es el trabajo creativo? está subsumido en el capital cultural o en el capital que entra en ese tipo de industria, ¿cuáles son las contradicciones sociales que están por detrás de esto? Esa es la cuestión.

\section{¿Un concepto como el de Audience commodity ${ }^{5}$ sigue vigente?}

Sí, totalmente. La Audience commodity es la gran forma de financiación de internet, o sea, la lógica de Google y Facebook es esa: la lógica de producción de Audiences commodity y de formas extremadamente sofisticadas, además, formas de creación de contacto directo con los individuos consumidores. Es una sofisticación de lo que nosotros conocimos como función publicitaria. Entonces, el concepto de Audience commodity es absolutamente central, el problema son las interpretaciones de esto que le dan ciertos autores como Christian Fuchs, al que le hice una crítica ${ }^{6}$, pues tiene una lectura de Marx en la que confunde producción y consumo como si fueran una misma cosa. En el caso de Fuchs es una adherencia a las ideas de Dallas Smythe, que es el autor de referencia, que es buenísimo; yo lo analicé de forma muy sobria, tiene una contribución fundamental, es el fundador del campo en Norteamérica, pero a ese respecto se equivocó completamente.

\section{¿Por decir que la audiencia trabaja?}

Claro que la audiencia trabaja, y luego tiene que inventar cuál es el trabajo de la audiencia. Que trabaja aprendiendo ¿aprendiendo a qué? a consumir dice; entonces esta es una confusión que luego no consigue explicar. Muchos lo han criticado, pero con internet este tema vuelve, porque la apariencia

5 En 1977 Dallas Smythe publicó en el Canadian Journal of political and social theory un artículo titulado

"Communications: Blindspot of western Marxism" en el que criticaba la mirada marxista sobre los medios de comunicación y en el que además acuñó el concepto de Audience commodity con el que buscaba explicar que el producto de los medios de comunicación de masas no eran los contenidos, sino las audiencias que eran vendidas como mercancías a los anunciantes.

6 Christian Fuchs es profesor de la Universidad Westminster que ha rescatado y defendido el pensamiento de Dallas Smythe dentro de la economía política. En años recientes Fuchs y Bolaño han sostenido una discusión en torno al concepto de trabajo en la era digital. Ver:Bolaño, César. (2015). Digitalization and labour: a rejoinder to Christian Fuchs. Triple C, 3 (1). Bolaño, César R. S. and Eloy S. Vieira. 2015. The Political Economy of the Internet: Social Networking Sites and a Reply to Fuchs. Television \& New Media 16 (1), 52-61.

Fuchs, Christian. (2015). Against Divisiveness: Digital Workers of the World Unite! A Rejoinderto César Bolaño and Eloy Vieira. Television\& New Media 16 (1), 62-71. 
de internet está más próxima a eso, porque internet significa que hay una actividad de la audiencia, exige actividad y esa actividad es muy fácil de entender como trabajo, porque ahí se puede decir que todos los que nos estamos divirtiendo en internet estamos trabajando, y con eso la lucha de clases se transforma en una lucha del capital y del imperio contra las multitudes, ya no hay distinción de clases, y es una explicación muy sencilla para gente que no ha leído a Marx. Por eso en el campo de la comunicación esto entra muy fácil. Además, es gente que tiene una lectura pésima y ni se dio cuenta de lo que significa la crítica de la Economía Política.

\section{¿Y los agujeros negros ${ }^{7}$ ya están tapados?}

No, yo creo que no, pero se avanzó mucho y la comunicación está en el centro del proceso. La situación actual en términos de pensamiento Marxista es hacer la crítica de la comunicación; ese fue mi proyecto de vida cuando escribí mi primer trabajo. Es, creo yo, el proyecto de la economía política de la comunicación y la cultura, es decir: nosotros tenemos que hacer lo que Marx ha hecho en el siglo xIx sobre la economía, ahora hay que hacer la crítica a la epistemología de la comunicación, y eso es lo difícil.
Uno de sus libros más importantes es Mercado Brasileño de Televisión ¿Cómo estudiar los mercados audiovisuales en la era digital?

Ese es un trabajo que mucha gente está haciendo, por ejemplo, yo sigo con el tema de la tv y me traslado a la ep de internet, son lógicas distintas; la lógica de internet es una lógica en ascensión que tiende a subsumir a la otra. La tv todavía es el mejor negocio para los capitales nacionales, pero hay una tendencia de subsunción y de superación de la industria cultural por internet. No es que desaparezca, es que se generaliza y llega a definir una lógica para todas las relaciones sociales. Es un avance de las formas de regulación mercantil de lo social. Yo estudio el tema de la tv ¿qué pasó con la tv brasileña? En este momento es bastante claro, qué ha hecho la Globo en relación al avance de las empresas telefónicas del país. Estos procesos hay que estudiarlos en términos microeconómicos, caso a caso, para entender de forma compleja lo que está pasando. Este es el proyecto en términos de análisis microeconómico que le toca también a la economía política de la comunicación.
7 Como "agujeros negros” se tradujo lo que Dallas Smythe denominó Blindspot con lo que hacía referencia a que los marxistas no estudiaban de los medios. Smythe afirmaba que los marxistas se concentraban en el análisis ideológico de los medios, dejando de lado la perspectiva económica. Las afirmaciones de Smythe abrieron un debate internacional en el que participaron diferentes autores. Ver: Artz, L. (2008). Media Relations and Media Product: Audience Commodity. Democratic Communiqué, 22 (1), 60-74.

Bolaño, C. (2006). Tapando el agujero negro. Para una crítica de la economía política de la comunicación. Cuadernos de Información y Comunicación, (11), pp. 47-53.

Jhally, S. (1982). Probing the blindspot: the audiende commodity. Canadian Journal of political and social theory, 6(1-2), 204-210.

Livant, B. (1979). The audience comodity: on the "blindspot" debate. Canadian Journal of political and social theory, 3(1), 91-106.

Murdock, G. (2006). Los agujeros negros del marxismo occidental: Respuesta a Dallas Smythe. Cuadernos de Información y Comunicación, (11), 11-22. 